\title{
Organizações Não Governamentais na área da Saúde da Criança - revisão da literatura
}

\author{
Non-Governmental Organizations in the area of Child Healthcare \\ - review of the literature
}

\author{
Ana Maria Aranha Magalhães Costa ${ }^{1}$ \\ Kátia Silveira da Silva ${ }^{1}$ \\ Cláudia Bonan ${ }^{1}$
}

${ }^{1}$ Instituto Fernandes Figueira, Fundação Oswaldo Cruz. Av. Rui Barbosa 716

Flamengo. 22250-020 Rio de Janeiro RJ.

magcosta@iff.fiocruz.br

\begin{abstract}
The second half of the twentieth century saw the emergence of organizations that reflected the mobilization of civil society for more effective participation in questions in the public interest and of a social nature. These became known as non-governmental organizations (NGOs) and they assumed their place as partners with public and private sectors to develop actions in different areas, including healthcare. Based on a review of the literature, the scope of this paper was to assess the status of scientific knowledge on the participation of NGOs in child healthcare and, by identifying and classifying their activities, establish if they are in line with the agenda of the field. Analysis of the publications took into consideration where the authors were from, the journal in question, the origins and area of activity of the NGOs and the source of the funds applied to the projects implemented. The nature of activities varied with the degree of development of the region where the NGOs operated and the themes, partnership between government and private sector agencies, sustainability and impact of actions on the favored communities were the main topics highlighted by the authors. The conclusion reached was that the performance of NGOs in child health is in the early stages and is a knowledge field to be explored.
\end{abstract}

Key words NGO, Healthcare, Children
Resumo A segunda metade do século vinte assistiu ao crescimento de entidades que refletiam a mobilização da sociedade civil para uma participação mais efetiva nas questões de interesse público e de cunho social. Desta forma surgiram as organizações não governamentais (ONG) que ganharam espaço como parceiros dos setores públicos e privados para a atuação em diferentes áreas, entre elas a saúde. Baseado em uma revisão da literatura, este trabalho teve como objetivo conhecer o status do conhecimento científico sobre a participação das ONG na saúde da criança e a partir da identificação e classificação de suas atividades, observar se esta atuação tem se compatibilizado com a agenda desse campo. A análise das publicações considerou a procedência dos autores, o periódico, os objetivos, a origem e o local de atuação das ONG e a procedência dos recursos aplicados nos projetos desenvolvidos. A natureza das atividades variou de acordo com o grau de desenvolvimento da região onde as ONG atuavam e os temas, parceria entre órgãos de governo e terceiro setor, sustentabilidade e impacto das ações nas comunidades beneficiárias foram os principais tópicos destacados pelos autores. Concluiu-se que as questões estão apenas se esboçando e que o impacto das intervenções das ONG na área da saúde da criança é um campo de conhecimento a ser explorado.

Palavras-chave ONG, Saúde, Criança 


\section{Introdução}

No rastro das profundas mudanças sociais, políticas, econômicas e culturais que recobriram as sociedades modernas e afetaram múltiplos sistemas de relações e práticas sociais, a partir da segunda metade do século XX, em várias partes do mundo, surgiram formas associativas com nova identidade que têm sido denominadas "organizações não governamentais” (ONG). Tal fenômeno não ficou restrito a países menos desenvolvidos e nem se deu exclusivamente em marcos nacionais. Dinâmicas transnacionais e internacionais impulsionaram a formação das ONG e muitas delas se fortaleceram em conexão com redes internacionais de ação social e política e de solidariedade, ganharam visibilidade em fóruns como as conferências das Nações Unidas, e têm sido financiadas ou apoiadas por agências internacionais.

No continente latino-americano, a formação de ONG teve grande impulso entre os anos de 1970 e 1980, quando segmentos da sociedade civil e movimentos sociais emergentes assumiram um papel central nos processos de redemocratização dos países da região e proliferaram iniciativas políticas em diversas esferas da vida social.

A conceitualização de "organização não governamental" não é tarefa simples. Nas últimas duas décadas, o termo ONG se difundiu e tem sido usado para denotar um conjunto heterogêneo de organizações. Para Landim ${ }^{1}$, ONG é uma categoria socialmente construída, inclusive pelas próprias organizações surgidas nesse período da história em busca de visibilidade e legitimidade. $\mathrm{O}$ termo adquire um caráter polissêmico, na medida em que é apropriado por diferentes atores sociais, em diferentes campos discursivos e ideológicos, e seu significado é disputado. Desse modo, para uma compreensão dessas novas modalidades de organização e ação é necessária uma análise que se debruce sobre a construção social de uma forma organizacional específica, ou de um conjunto de organizações que vêm ocupando posições análogas no espaço social, nas duas últimas décadas ${ }^{1}$.

As ONG diferenciam-se de partidos políticos, igrejas, sindicatos, associações comunitárias e universidades, apesar das interseções históricas com essas entidades e de elas terem sido a origem de muitos de seus membros. Os analistas têm sistematizado uma série de características peculiares dessas novas formas associativas: possuem uma irredutível dimensão política, embora não tenham cunho partidário; rompem com a lógica filantrópica e a noção de caridade das entidades religiosas, e atuam na perspectiva do fortalecimento da cidadania (justiça social, direitos, empoderamento e participação); investem na produção de conhecimento e na formação de seus quadros, para fins de sustentação de sua intervenção, sem se confundirem com instituições acadêmicas; de modo diferente de entidades sindicais e comunitárias, não pretendem ser "representativas" de uma coletividade maior, mas valorizam a proliferação de várias organizações atuando na mesma área de maneira articulada, preservando cada uma sua autonomia, e o incremento da participação direta e qualificada da sociedade nos espaços de debate e decisão política e planejamento social; enfim, apesar de seu caráter não governamental, não recusam a interlocução com o Estado e as instituições políticas, com os quais podem manter relações de conflito ou colaboração ${ }^{1,2}$.

As ONG têm atuado de modos distintos, como a prestação de serviços, o estímulo a organização de segmentos da sociedade, a proposição de projetos de lei ou de políticas públicas, a mobilização em torno de direitos difusos e específicos e a construção de modos alternativos de produção, visando o desenvolvimento social e o fortalecimento da cidadania ${ }^{3,4}$. Uma maior percepção da sociedade como co-partícipe do desenvolvimento social faz com que ela traga para si compromissos que outrora eram vistos apenas como responsabilidades de governos. Ao mesmo tempo, grupos organizados conferem maior legitimidade às reivindicações e aumentam o poder de pressão sobre as autoridades públicas. Entretanto, as relações entre ONG e Estado e a definição de seus respectivos papéis na promoção do desenvolvimento social e dos direitos têm sido permeadas por ambiguidades e contradições, e são objeto de discussões acadêmicas e políticas. Por um lado, na perspectiva da equidade, da inclusão e da justiça social debatese a conveniência e os riscos da transferência de atribuições e responsabilidades que tradicionalmente foram (ou deveriam ter sido) do Estado à sociedade (ideia que tem defensores e críticos acirrados). Por outro lado, a experiência das ONG é vista também a partir de seu potencial de ampliar o horizonte democrático, permitindo novas formas de participação social e política, fomentando o surgimento de novos sujeitos, temáticas e direitos, e aprofundando um projeto de cidadania inclusivo e pluralista.

A assunção de compromissos e responsabilidades públicas por parte das ONG coloca ainda a questão da sustentabilidade, ao longo do tempo, das ações desenvolvidas por elas, inclusive 
no sentido financeiro. Por definição, as ONG são entidades sem fins lucrativos e sem prerrogativas tributárias, portanto, sua capacidade de autofinanciamento é extremamente limitada. Para desenvolver e implementar projetos, dependem de parcerias com setores públicos e/ou privados e de acesso a seus fundos. A dotação de recursos financeiros é voluntária, ou seja, não há obrigatoriedade legal de destinação e continuidade desses financiamentos. Esses fatos tornam ainda mais complexas e desafiadoras não somente as relações entre essas entidades e o Estado, mas as relações triangulares entre Estado, mercado e instituições da sociedade civil.

De modo oportuno, Landim ${ }^{5}$ observa que os significados e os papéis das entidades associativas em geral, inclusive das ONG, não podem ser compreendidos fora do contexto histórico contemporâneo, marcado por transformações profundas - e ainda em curso - do Estado nacional e das relações globais, da política e da economia, do mundo do trabalho, dos laços sociais e das formas de solidariedade, pelo aumento da vulnerabilidade e da fragmentação social, pela descentralização político-administrativa.

Entre os diversos setores de planejamento social e intervenção política em que se dá a presença das ONG está o da saúde. No Brasil e na América Latina, a participação dessas organizações no campo da saúde tem sido objeto de estudos que têm se interrogado acerca de muitas questões: seu papel no aprofundamento do marco jurídico-normativo dos direitos sanitários e sua capacidade política e técnica de influenciar no desenho e na implementação de programas; seu potencial para incrementar o controle social e a participação da sociedade nas decisões sobre políticas de saúde; o impacto de suas atividades sobre as condições de vida e saúde; os desafios do desenvolvimento institucional, da mobilização de recursos e da sustentabilidade financeira das ONG e de seus projetos; enfim, sobre possíveis tendências e significados dessas organizações no curso das mudanças nas relações entre Estado e sociedade ${ }^{4,6-9}$.

Há uma grande concentração de estudos sobre a atuação das ONG em áreas como as da AIDS e da saúde e direitos reprodutivos no Brasil, refletindo, de certa forma, o forte ativismo em torno desses temas e a legitimidade pública e institucional conquistada por agendas comuns construídas nos circuitos do sistema internacional e nacional ${ }^{2}$. Entretanto, interroga-se o quanto se tem acumulado de conhecimentos e reflexões acerca da atuação das ONG em outras áre- as de saúde que também têm sido lócus de ativismo e de participação social, como a saúde infantil. Com base em uma revisão da literatura nacional e internacional, o objetivo deste estudo foi descrever a produção científica sobre a atuação das ONG na área da saúde da criança, examinando mais especificamente: a) o que se conhece das atividades desenvolvidas por essas organizações, como se articulam com as agendas globais e nacionais e como são avaliadas; b) quais são as questões teórico-conceituais e políticas que essa literatura problematiza e como dialoga com o campo mais vasto dos estudos sobre ONG.

\section{Método}

Foi realizada uma revisão da produção científica disponível sobre as ações das ONG na área de saúde da criança, tendo como fontes de informação os bancos de dados do Medline, referente à National Library of Medicine (EUA ${ }^{10}$, e do Lilacs (Literatura Latino-americana e do Caribe em Ciências da Saúde) ${ }^{11}$, além de busca manual de artigos referenciados nessas publicações. Foram levantados todos os artigos publicados até 31 de dezembro de 2007.

Os descritores utilizados para a busca foram: organização não governamental ou ONG (No Governmental Organization ou NGO); criança ou crianças (child ou children); saúde ou doença (health ou disease); morbidade (morbidity) e mortalidade (mortality). O processo de busca e seleção de artigos foi condicionado pelo conceito de ONG adotado nos Descritores em Ciência da Saúde (BVS)12: "instituições da sociedade civil, geralmente fundações ou associações civis sem fins lucrativos, com finalidades de interesse público”.

Para a inclusão no estudo, os artigos tiveram que cumprir dois critérios: referência textual a organizações não governamentais com atuação em qualquer área da saúde voltada para a criança e ter sido escrito nos idiomas inglês, português, espanhol, francês ou italiano. Foram excluídos os artigos cujas ações eram dirigidas exclusivamente à saúde da mulher, mesmo que estas tivessem algum reflexo sobre a saúde da criança. Os artigos que não estavam disponíveis em bibliotecas nacionais também foram excluídos.

Para descrição dos artigos foram considerados os seguintes aspectos: nome e catalogação da revista; ano de publicação do artigo e procedência institucional do autor principal; objetivos do artigo; abordagem metodológica; principais resultados e discussões. 
Em relação, aos tópicos inerentes às $\mathrm{ONG}$ citadas nesses artigos, foram descritas as seguintes características: local de atuação e de origem geográfica das ONG; procedência dos recursos de fomento aos projetos das ONG; atividades desenvolvidas pelas ONG. Estas atividades foram categorizadas previamente como: a) pesquisa e/ou coleta de dados; b) participação na agenda das políticas e programas de saúde da criança; c) assistência à saúde e ao bem estar, incluindo promoção, prevenção e tratamento; d) capacitação de profissionais de saúde; e) ações de educação e informação em saúde dirigidas a membros da comunidade; f) provisão e gestão de recursos de captação de parcerias (Quadro 1).

\section{Resultados}

A busca nas bases de dados Medline ${ }^{10}$ e Lilacs $^{11}$ com os descritores selecionados resultou em 357 e 24 artigos respectivamente. Após revisão dos resumos, apenas em 178 artigos foram identificadas ações de ONG na área de saúde maternoinfantil. Deste total, foram selecionados 86 artigos que se concentravam em ações dirigidas à população infantil; os demais descreviam ações voltadas para a saúde da mulher, tais como planejamento familiar. Após a aplicação dos outros critérios de inclusão e de exclusão obteve-se 57 artigos (Figura 1).

Quadro 1. Descrição das atividades desenvolvidas por ONG na área de saúde da criança.

\begin{tabular}{|l|l|}
\hline \multicolumn{1}{|c|}{ Atividade } & \multicolumn{1}{c|}{ Descrição } \\
\hline Pesquisa (P) & $\begin{array}{l}\text { Atividades relacionadas a levantamento e avaliação de indicadores de } \\
\text { programas e/ou práticas em saúde (P1) } \\
\text { Estudos de prevalência (P2) }\end{array}$ \\
\hline $\begin{array}{l}\text { Participação na agenda } \\
\text { programática e política } \\
\text { (PAP) }\end{array}$ & $\begin{array}{l}\text { Colaboração nas discussões de políticas para a saúde da criança (PAP1) } \\
\text { Definição de calendários e de agendas (PAP2) } \\
\text { Participação em manifestos e workshops (PAP3) } \\
\text { Apoio na vigilância do cumprimento de normas (PAP4) }\end{array}$ \\
\hline $\begin{array}{l}\text { Assistência à saúde e ao } \\
\text { bem estar (ASBE) }\end{array}$ & $\begin{array}{l}\text { Participação em programas de saúde: atenção básica, vacinação, crescimento } \\
\text { e desenvolvimento/ nutrição, reidratação oral (ASBE1) } \\
\text { Atendimento à criança portadora de câncer, doenças infecciosas ou outras } \\
\text { patologias (ASBE2) } \\
\text { Assistência à criança portadora de deficiência (ASBE3) } \\
\text { Assistência à criança de rua / vítima de violência (ASBE4) } \\
\text { Assistência à criança órfã / refugiada de guerra (ASBE5) }\end{array}$ \\
\hline $\begin{array}{l}\text { Capacitação (C) } \\
\text { Treinamento de profissionais e de agentes de saúde (C1) }\end{array}$ \\
\hline $\begin{array}{l}\text { Educação e informação } \\
\text { em saúde (EIS) }\end{array}$ & $\begin{array}{l}\text { Orientação de pais e familiares sobre cuidados de saúde, identificação e } \\
\text { prevenção de doenças (EIS1) } \\
\text { Educação sexual de crianças e de adolescentes (EIS2) } \\
\text { Orientação de jovens na prevenção de drogas (EIS3) } \\
\text { Divulgação de informações sobre temas da saúde da criança através de sítios } \\
\text { da Internet (EIS4) }\end{array}$ \\
\hline $\begin{array}{l}\text { Suporte material (PGR1) } \\
\text { Estabelecimento de contatos para captação de provedores e parceiros (PGR2) } \\
\text { Gestão de hospitais, clínicas, centros de saúde e programas (PGR3) }\end{array}$ \\
\hline
\end{tabular}

\begin{tabular}{|c|c|c|c|c|c|c|}
\hline $\begin{array}{l}\text { Busca eletrônica } \\
\text { Medline } \\
\text { Lilacs }\end{array}$ & $\rightarrow$ & $\begin{array}{c}\text { Identificação } \\
160 \text { artigos /Medline } \\
18 \text { artigos/ Lilacs }\end{array}$ & $\rightarrow$ & $\begin{array}{c}\text { Selecionados } \\
72 \text { artigos/Medline } \\
14 \text { artigos/ Liliacs }\end{array}$ & $\rightarrow$ & $\begin{array}{c}\text { Inclusão } \\
51 \text { artigos/Medline } \\
6 / \text { Lilacs }\end{array}$ \\
\hline
\end{tabular}

Figura 1. Representação esquemática de busca e resultados. 
As publicações abrangeram o período de 1987 a 2007, destacando-se o ano de 2004 com 08 artigos. Em 2005, 2006 e 2007, a produção foi de 06 artigos por ano. Comparando-se os anos $90 \mathrm{com}$ a primeira década do ano 2000, observa-se um aumento superior a 50\% no número de publicações. A consolidação do termo ONG, a partir de meados dos anos 80, talvez explique a inexistência, nos bancos de dados consultados, de produções anteriores a 1987. Entre os artigos selecionados não foram encontradas publicações nos anos de 88, 89, 94 e 95.

Os artigos foram publicados em 47 revistas da área temática de ciências da saúde, catalogadas pela Biblioteca Virtual de Saúde ${ }^{12}$ como: especialidades médicas (23 revistas); ocupações em saúde, que abrange os assuntos de nutrição, enfermagem e odontologia (06); população e saúde, que aborda os temas de administração, epidemiologia, saúde pública (08); e classificados como "outros" os temas educação, medicina esportiva, reabilitação, medicina tropical (08). Dois periódicos não foram localizados no catálogo da Biblioteca Virtual de Saúde.

Cinquenta e uma publicações do acervo foram editadas em revistas estrangeiras enquanto as nacionais contribuíram com seis artigos. O periódico Health Policy and Planning publicou o tema com maior frequência editando 05 artigos $^{13-}$ ${ }^{17}$ seguido do International Journal of Health Planning and Management com três ${ }^{18-20}$ e dos Journal Community Health ${ }^{21,22}$, Journal Health Population Nutrition ${ }^{23,24}$, e Social Science and Medicine ${ }^{25,26}$ cada um, com 2 artigos. Os demais periódicos apresentaram apenas uma publicação.

O conhecimento sobre o tema se produziu, basicamente, em instituições acadêmicas. As universidades, instituições de ensino e centros de pesquisa foram a origem dos autores principais de aproximadamente metade dos artigos (58\%). Os demais foram elaborados por profissionais provenientes de ONG (16\%), organismos internacionais (12\%), órgãos governamentais (07\%), serviços de saúde $(02 \%)$, indústria farmacêutica (02\%) e empresa de consultoria (03\%), sendo esta última prestadora de serviços à $\mathrm{ONG}$ de grande porte.

Seis temas centrais poderiam sintetizar a diversidade de objetos e objetivos de investigação dos artigos (Tabela 1).

Conforme veremos mais adiante, somente cerca de $60 \%$ desses artigos se dedicam, em alguma medida, a análise da atuação de ONG em ações de saúde infantil. Nos demais artigos, em que pese seu objetivo de discutir temas relacionados à saúde da criança, as ONG são apenas citadas.

A heterogeneidade dos objetivos dos artigos justifica a diversidade de métodos adotados para atingi-los. Foram identificados 24 artigos baseados em pesquisa qualitativa, incluindo estudo de casos e revisão bibliográfica. Um total de 32 artigos apresentou resultados de estudos epidemiológicos, como estudos transversais e caso-controle. Apenas 1 estudo utilizou combinadamente métodos qualitativos e quantitativos.

As atividades desenvolvidas por ONG na área de saúde da criança, descritas ou mencionadas nesses artigos, foram classificadas de acordo com critérios previamente definidos no Quadro 1.

No acervo coletado pela pesquisa, $75 \%$ dos artigos apresentaram ONG desenvolvendo uma única atividade e $25 \%$ relataram uma conjugação de intervenções. Observou-se um predomínio das ações relacionadas à área da assistência à saúde e ao bem estar, sendo elas identificadas em 61\% dos artigos (Quadro 2). A gestão de recursos e a captação de parceria foram descritas em 21\% das publicações, atividades de educação e informação foram observadas em 19\%, a participação na agenda programática e política em $16 \%$, a capacitação em $12 \%$, e a pesquisa em 5\%

Tabela 1. Temas centrais identificados nos artigos selecionados.

\begin{tabular}{lr}
\hline \multicolumn{1}{c}{ Temas } & $\begin{array}{c}\text { No de artigos } \\
(\%)\end{array}$ \\
\hline Estratégias e ações para a melhoria das condições de saúde infantil & $35(61 \%)$ \\
Aspectos epidemiológicos ou fatores de risco para patologias especificas & $7(12 \%)$ \\
Custos ou recursos empregados em ações de saúde e bem estar da população infantil & $6(11 \%)$ \\
Processo de negociação política na área de saúde da criança & $4(7 \%)$ \\
Parcerias entre ONG e outras instituições & $3(5 \%)$ \\
Sustentabilidade de projetos desenvolvidos por ONG que atuam na área de saúde da criança & $2(4 \%)$ \\
\hline
\end{tabular}




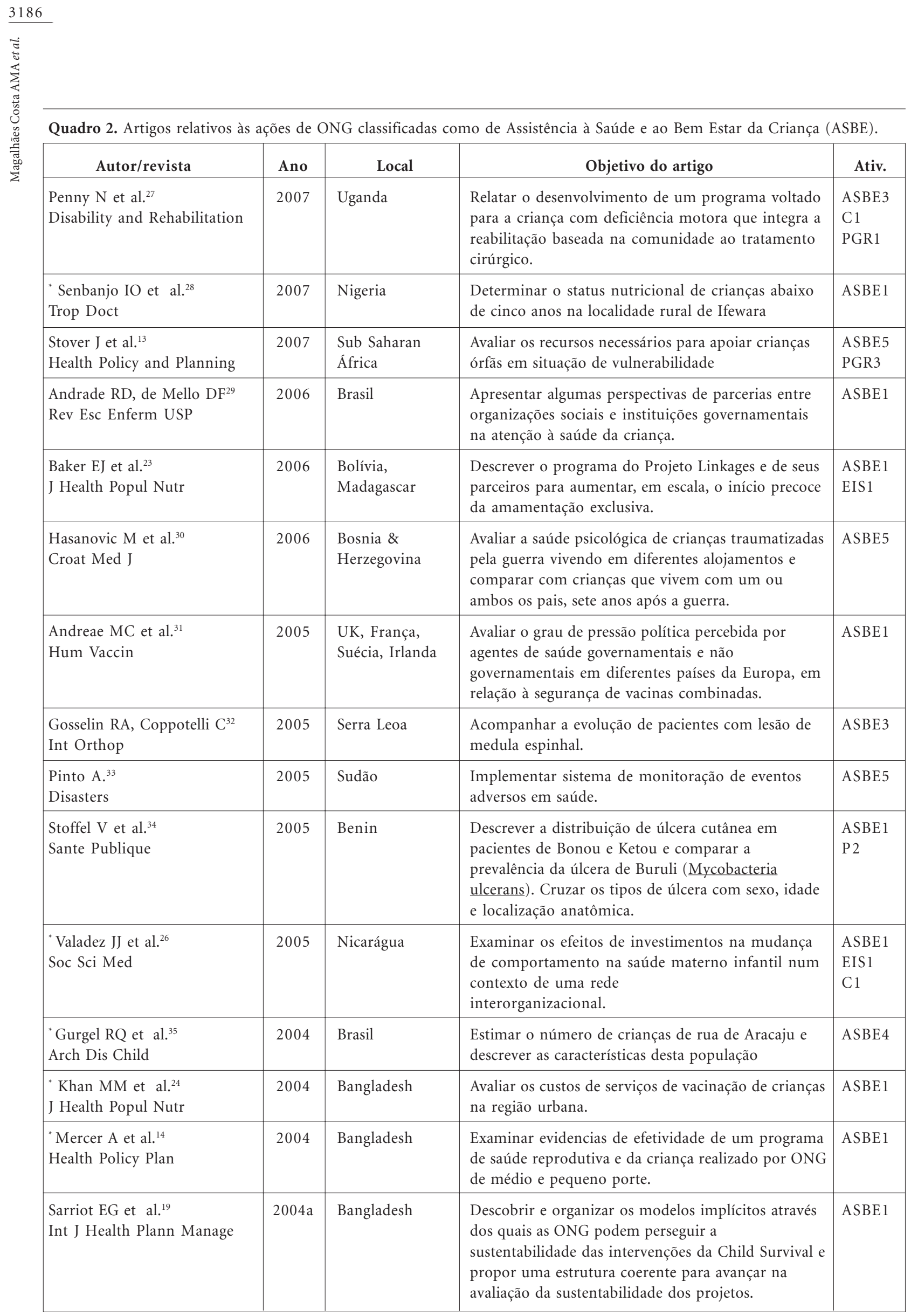


Quadro 2. continuação

\begin{tabular}{|c|c|c|c|c|}
\hline Autor/revista & Ano & Local & Objetivo do artigo & Ativ. \\
\hline $\begin{array}{l}\text { Sarriot, EG et al. }{ }^{20} \\
\text { Int J Health Plann Manage }\end{array}$ & $2004 \mathrm{~b}$ & Bangladesh & $\begin{array}{l}\text { Avaliar, através da metodologia CSSA (Child Survival } \\
\text { Sustainability Assessment) a sustentabilidade do } \\
\text { cuidado primário em saúde desenvolvido por ONG e } \\
\text { por projetos baseados na comunidade. }\end{array}$ & ASBE 1 \\
\hline $\begin{array}{l}\text { de Souza AI, Erdmann } \text { AL }^{36} \\
\text { Rev Gaúcha Enferm }\end{array}$ & 2003 & Brasil & $\begin{array}{l}\text { Refletir sobre algumas implicações sociais e políticas } \\
\text { que o cuidado à criança com câncer e sua família } \\
\text { demanda. }\end{array}$ & ASBE2 \\
\hline $\begin{array}{l}\text { Sperandio } \mathrm{AMG}^{37} \\
\text { Rev Bras Cresc Desenv Hum }\end{array}$ & 2003 & Brasil & $\begin{array}{l}\text { Descrever os passos da estratégia para construção e } \\
\text { implantação de um projeto que envolveu ações de } \\
\text { promoção e proteção da saúde ocular para crianças } \\
\text { de } 0 \text { a } 07 \text { anos no município de Morungaba, SP de } \\
2000 \text { a } 2003 \text {. }\end{array}$ & ASBE3 \\
\hline $\begin{array}{l}\text { Winch PJ et al. }{ }^{15} \\
\text { Health Policy Plan }\end{array}$ & 2002 & & $\begin{array}{l}\text { Descrever o terceiro componente da estratégia de } \\
\text { manejo integrado pela família e pela comunidade, } \\
\text { das doenças da infância (IMCI). }\end{array}$ & $\begin{array}{l}\text { ASBE1 } \\
\text { PAP3 }\end{array}$ \\
\hline $\begin{array}{l}{ }^{*} \text { Akerman } \mathrm{M} \text { et } \text { al. }^{38} \\
\text { Arq Med ABC }\end{array}$ & 2001 & Brasil & $\begin{array}{l}\text { Caracterizar a atuação da ONG CRAMI-ABCD e } \\
\text { propor medidas de prevenção à violência infantil. }\end{array}$ & ASBE4 \\
\hline $\begin{array}{l}\text { Morikawa } \mathrm{M}^{39} \\
\text { J Trop Pediatr }\end{array}$ & 2001 & Kosovo & $\begin{array}{l}\text { Examinar os fatores de risco para infecções } \\
\text { respiratórias agudas em crianças atendidas na } \\
\text { emergência durante o conflito interno no Kosovo. }\end{array}$ & $\begin{array}{l}\text { ASBE1 } \\
\text { PGR1 }\end{array}$ \\
\hline $\begin{array}{l}\text { Salvarani MCC, Decesaro M } \\
\text { das } \mathrm{N}^{40} \\
\text { Nursing }\end{array}$ & 2001 & Brasil & $\begin{array}{l}\text { Traçar o perfil da população de famílias } \\
\text { acompanhadas pela Pastoral da Criança em Maringá } \\
\text { e Sarandi (PR), identificar a população atendida na } \\
\text { Casa de Recuperação da Criança Desnutrida CRD, } \\
\text { identificar as dificuldades da proposta de trabalho da } \\
\text { CRD e definir o papel do enfermeiro na recuperação } \\
\text { da criança desnutrida }\end{array}$ & ASBE 1 \\
\hline $\begin{array}{l}\text { Parrilla Rodríguez AM, } \\
\text { Gorrin Peralta JJ } \\
\text { P R Health Sci J }\end{array}$ & 2000 & Porto Rico & $\begin{array}{l}\text { Discutir aspectos éticos nas promoções do } \\
\text { aleitamento humano e da amamentação. }\end{array}$ & $\begin{array}{l}\text { ASBE1 } \\
\text { EIS1 } \\
\text { PAP3 } \\
\text { PAP4 }\end{array}$ \\
\hline $\begin{array}{l}\text { Ranganathan K et al. }{ }^{42} \\
\text { Oral Dis }\end{array}$ & 2000 & Índia & $\begin{array}{l}\text { Descrever a apresentação clínica e a prevalência de } \\
\text { lesões orais, em } 300 \text { pacientes de uma ONG, HIV } \\
\text { positivos e sintomáticos, por um período de nove } \\
\text { meses. }\end{array}$ & ASBE2 \\
\hline $\begin{array}{l}\text { Rajeswari R et al. }{ }^{43} \\
\text { Int J Tuberc Lung Dis }\end{array}$ & 1999 & Índia & $\begin{array}{l}\text { Quantificar o impacto sócio econômico da } \\
\text { tuberculose em pacientes e suas famílias em áreas } \\
\text { rural e urbana. }\end{array}$ & ASBE2 \\
\hline $\begin{array}{l}\text { Wegman } \mathrm{ME}^{44} \\
\text { Pediatrics }\end{array}$ & 1999 & & $\begin{array}{l}\text { Fazer uma revisão sobre saúde da criança no mundo } \\
\text { e os tratamentos disponíveis, identificar as ajudas } \\
\text { internacionais, levantar questões sobre o trabalho } \\
\text { infantil, convenção das nações unidas e direitos da } \\
\text { criança. }\end{array}$ & ASBE 1 \\
\hline $\begin{array}{l}\text { Condamine JL et al. }{ }^{45} \\
\text { Med Trop }\end{array}$ & 1998 & Burkina Faso & $\begin{array}{l}\text { Apresentar os princípios e a organização do centro } \\
\text { de reeducação e de aparelhagem para deficientes } \\
\text { motores, analisando prontuários e discutindo o } \\
\text { acompanhamento dos pacientes deste centro. }\end{array}$ & ASBE3 \\
\hline
\end{tabular}




\begin{tabular}{|c|c|c|c|c|c|}
\hline \multirow{10}{*}{ 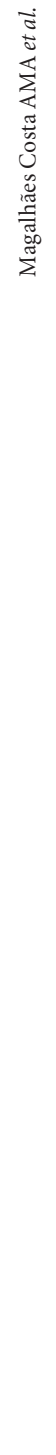 } & Quadro 2 . continuação & & & & \\
\hline & Autor/revista & Ano & Local & Objetivo do artigo & Ativ. \\
\hline & $\begin{array}{l}\text { Pal DK et al. }{ }^{46} \\
\text { Int J Epidemiol }\end{array}$ & 1998 & & $\begin{array}{l}\text { Comparar a sensibilidade, eficácia e custos entre } \\
\text { métodos para acompanhamento de criança com } \\
\text { epilepsia. }\end{array}$ & ASBE2 \\
\hline & $\begin{array}{l}{ }^{*} \text { Amin R, Li Y Y } \\
\text { Women Health }\end{array}$ & 1997 & Bangladesh & $\begin{array}{l}\text { Examinar o impacto de programas de crédito } \\
\text { promovidos por ONG na mortalidade infantil e na } \\
\text { cobertura vacinal na zona rural. }\end{array}$ & $\begin{array}{l}\text { ASBE1 } \\
\text { EIS } 1\end{array}$ \\
\hline & $\begin{array}{l}\text { Leifert } \mathrm{H}, \text { Murahovsch } \mathrm{J}^{48} \\
\text { Pediatria Moderna }\end{array}$ & 1997 & Brasil & $\begin{array}{l}\text { Detectar e resolver problemas de visão do maior } \\
\text { número possível de crianças carentes de } 06 \text { a } 13 \\
\text { anos da periferia de cidade de São Paulo, sem ônus } \\
\text { para a família e para o Estado. }\end{array}$ & ASBE3 \\
\hline & $\begin{array}{l}\text { van der Stuyft P49 } \\
\text { Ann Trop Med Parasitol }\end{array}$ & 1997 & Guatemala & $\begin{array}{l}\text { Investigar padrões de utilização de serviços de saúde } \\
\text { nas doenças materno-infantis, avaliar as despesas } \\
\text { que incidem sobre os diferentes provedores destes } \\
\text { serviços. }\end{array}$ & ASBE1 \\
\hline & $\begin{array}{l}\text { Gellert GA }{ }^{18} \\
\text { Int J Health Plann Manage }\end{array}$ & 1996 & & $\begin{array}{l}\text { Prover uma vaisão do espectro de atividades e } \\
\text { filosofias que caracterizam ações de ONG através da } \\
\text { descrição de algumas destas entidades que ocupam } \\
\text { posição de liderança. }\end{array}$ & $\begin{array}{l}\text { ASBE2 } \\
\text { EIS1 } \\
\text { PRG1 }\end{array}$ \\
\hline & $\begin{array}{l}\text { Thomas } \mathrm{M}^{50} \\
\text { Indian J Pediatr }\end{array}$ & 1992 & Índia & $\begin{array}{l}\text { Apresentar as experiências de parcerias em } \\
\text { programas da "Action Aid" de identificação e } \\
\text { intervenção precoce voltados para a criança } \\
\text { portadora de deficiência. }\end{array}$ & $\begin{array}{l}\text { ASBE3 } \\
\text { C1 } \\
\text { EIS1 }\end{array}$ \\
\hline & $\begin{array}{l}\text { Crane SF, Carswell JW51 } \\
\text { Health Educ Res }\end{array}$ & 1992 & & $\begin{array}{l}\text { Revisar e acessar os projetos de ONG na educação e } \\
\text { prevenção da AIDs para grupos marginalizados. }\end{array}$ & ASBE4 \\
\hline & $\begin{array}{l}{ }^{*} \text { Auer } C^{52} \\
\text { Southeast Asian J Trop Med } \\
\text { Public Health }\end{array}$ & 1990 & Filipinas & $\begin{array}{l}\text { Avaliar o status de saúde, especialmente das crianças, } \\
\text { em uma área ocupada da cidade. }\end{array}$ & $\begin{array}{l}\text { ASBE1 } \\
\text { C1 } \\
\text { PGR1 }\end{array}$ \\
\hline
\end{tabular}

*Artigos que analisam especificamente a atuação de ONG na área da saúde infantil.

(Quadro 3). Dos 14 artigos que apresentaram ONG desenvolvendo mais de uma atividade, 57\% associaram assistência e educação e/ou capacitação ${ }^{18,23,26,27,41,47,50,52}$, ações que se mostraram complementares. O treinamento de líderes e membros da comunidade e de familiares em práticas de cuidado da criança - preparação de solução oral, práticas de amamentação - assim como a promoção de atualização de profissionais de saúde, foram as ações mais frequentemente associadas à assistência.

Este conjunto de artigos revelou diferenças no que diz respeito às atividades desenvolvidas por ONG, segundo regiões mais ou menos desenvolvidas. Nas publicações referentes a países desenvolvidos, as organizações apareceram atuando principalmente nas esferas deliberativas, com assentos em fóruns de debate como representante de grupos sociais que reivindicam implementos ou mudanças na condução de programas de prevenção e promoção de saúde ${ }^{53-56}$. Nas publicações relacionadas aos países em desenvolvimento, as atividades predominantes foram aquelas voltadas para a assistência à saúde com características preventivas e ênfase na atenção básica: programas de imunização, nutrição, crescimento e desenvolvimento infantil ${ }^{14,15,23,24,28,40,44,47,52}$. Descreveram também atividades de reabilitação ${ }^{32,45} \mathrm{e}$ detecção precoce de sinais e sintomas de deficiências auditiva e neurológica ${ }^{50}$.

Cerca de 64 ONG foram citadas nominalmente em 34 artigos. Dentre elas, 38 eram de origem nacional e atuação local, sendo 15 dos Estados Unidos, 07 do Brasil, 06 de Bangladesh, 06 da Índia e uma do Kosovo, do México, da Nicarágua e do Sri Lanka. As demais eram ONG de porte internacional e atuavam em regiões em desenvolvimento, sendo de diversas origens, en- 
Quadro 3. Artigos relativos às ações de ONG classificadas como Pesquisa, Participação na Agenda Programática e Política, Capacitação, Educação e Informação em Saúde e Provedor e Gestor de Recursos e Captação de Parcerias

\begin{tabular}{|c|c|c|c|c|}
\hline Autor/revista & Ano & Local & Objetivo do artigo & Ativ. \\
\hline $\begin{array}{l}\text { Forsyth R et al. }{ }^{57} \\
\text { Dev Med Child Neurol }\end{array}$ & 2007 & UK & $\begin{array}{l}\text { Explorar um modelo quantitativo para avaliar a } \\
\text { influência do ambiente na participação da criança } \\
\text { portadora de deficiência neurológica. }\end{array}$ & PGR1 \\
\hline $\begin{array}{l}\text { Goren } S^{58} \\
\text { JCAPN-Journal of Child and } \\
\text { Adolescent Psychiatric Nursing }\end{array}$ & 2007 & Vietnam & $\begin{array}{l}\text { Avaliar a assistência prestada à saúde mental de } \\
\text { crianças e adolescentes e treinar profissionais para } \\
\text { estes cuidados. }\end{array}$ & PGR2 \\
\hline $\begin{array}{l}\text { Paulson JA, Arnesen SJ }{ }^{59} \\
\text { Pediatr Clin N Am }\end{array}$ & 2007 & world wide web & $\begin{array}{l}\text { Apresentar o uso da internet como um meio de } \\
\text { informação sobre saúde da criança. }\end{array}$ & EIS4 \\
\hline $\begin{array}{l}\text { Caraher M et al. }{ }^{53} \\
\text { Public Health Nutr }\end{array}$ & 2006 & $\begin{array}{l}\text { USA, Australia, } \\
\text { Europa }\end{array}$ & $\begin{array}{l}\text { Conduzir uma revisão das políticas de regulação da } \\
\text { publicidade de alimentos }\end{array}$ & PAP1 \\
\hline $\begin{array}{l}\text { Lechtig A et al. }{ }^{60} \\
\text { Food Nutr Bull }\end{array}$ & 2006 & Peru & $\begin{array}{l}\text { Apresentar a lição aprendida com a suplementação } \\
\text { de micro-nutrientes como componente do } \\
\text { Programa Integrado de Segurança Alimentar }\end{array}$ & PAP 1 \\
\hline $\begin{array}{l}\text { Rocha } \mathrm{PR}^{36} \\
\text { Rev Ter Ocup Univ São Paulo }\end{array}$ & 2006 & Brasil & $\begin{array}{l}\text { Apresentar a experiência de uma educadora social, } \\
\text { na implantação e implementação de um } \\
\text { equipamento para crianças e adolescentes em } \\
\text { situação de risco pessoal e social. }\end{array}$ & PGR \\
\hline $\begin{array}{l}\text { Potts-Datema W et al. }{ }^{56} \\
\text { Promot Educ }\end{array}$ & 2005 & USA & $\begin{array}{l}\text { Descrever a parceria entre ONG de atuação na saúde } \\
\text { pública e na educação e destacar estratégias de } \\
\text { sucesso. }\end{array}$ & PAP2 \\
\hline $\begin{array}{l}\text { Ahmed A et al. }{ }^{51} \\
\text { Prehospital Disaster Med }\end{array}$ & 2004 & Afeganistão & $\begin{array}{l}\text { Prover informações de linha de base para um } \\
\text { programa comunitário de saúde materno infantil. }\end{array}$ & $\mathrm{P} 1$ \\
\hline $\begin{array}{l}\text { Brusamolino E, Maffi G }{ }^{61} \\
\text { Crit Rev Oncol Hematol }\end{array}$ & 2004 & Costa do Marfim & $\begin{array}{l}\text { Rever criticamente uma experiência de cooperação } \\
\text { em saúde entre um hospital italiano de ensino e } \\
\text { pesquisa e um hospital africano. }\end{array}$ & $\begin{array}{l}\text { PGR1 } \\
\text { PGR2 } \\
\text { PGR3 }\end{array}$ \\
\hline $\begin{array}{l}\text { Cho H, Nadow } \mathrm{MZ}^{22} \\
\text { J Community Health }\end{array}$ & 2004 & USA & $\begin{array}{l}\text { Investigar as barreiras relativas à qualidade da } \\
\text { alimentação e a educação nutricional do Programa } \\
\text { Coordenado de Saúde Escolar. }\end{array}$ & PRG1 \\
\hline $\begin{array}{l}\text { Frenkel LD, Nielsen } \mathrm{K}^{55} \\
\text { Ann Allergy Asthma Immunol }\end{array}$ & 2003 & USA & $\begin{array}{l}\text { Rever e discutir aspectos relevantes da vacinação e da } \\
\text { imunização. }\end{array}$ & PAP2 \\
\hline $\begin{array}{l}\text { Balinska } \mathrm{MA}^{62} \\
\text { Lancet Infect Dis }\end{array}$ & 2003 & & $\begin{array}{l}\text { Apresentar a discussão do Workshop Internacional } \\
\text { sobre "vacinação na sociedade de amanhã". }\end{array}$ & PAP3 \\
\hline $\begin{array}{l}{ }^{*} \text { Babalola } S \text { et al. }{ }^{21} \\
\text { J Community Health }\end{array}$ & 2001 & Camarões & $\begin{array}{l}\text { Analisar o impacto da mobilização da comunidade, } \\
\text { em uma região urbana e outra rural, da promoção do } \\
\text { conhecimento e práticas no planejamento familiar, } \\
\text { DSTs e tratamento das doenças comuns da infância. }\end{array}$ & EIS1 \\
\hline $\begin{array}{l}\text { Chakraborty S et al. }{ }^{16} \\
\text { Health Policy Plan }\end{array}$ & 2000 & Índia & $\begin{array}{l}\text { Testar a efetividade de dois métodos para a melhoria } \\
\text { da qualidade da assistência prestada por práticos } \\
\text { privados à criança doente. }\end{array}$ & $\mathrm{P} 1$ \\
\hline $\begin{array}{l}\text { Pick S et al. }{ }^{63} \\
\text { Reprod Health Matters }\end{array}$ & 2000 & México & $\begin{array}{l}\text { Apresentar o trabalho de uma ONG mexicana - } \\
\text { IMIFAP - em programas de educação sexual para } \\
\text { crianças do pré-escolar à adolescência e a seus pais. }\end{array}$ & $\begin{array}{l}\text { C1 } \\
\text { EIS1 }\end{array}$ \\
\hline $\begin{array}{l}{ }^{*} \text { Aikins MK et al. }{ }^{25} \\
\text { Soc Sci Med }\end{array}$ & 1998 & Gambia & $\begin{array}{l}\text { Analisar o custo efetividade da implantação do } \\
\text { programa de mosqueteiros impregnados com } \\
\text { inseticida na prevenção da malária. }\end{array}$ & PRG1 \\
\hline
\end{tabular}




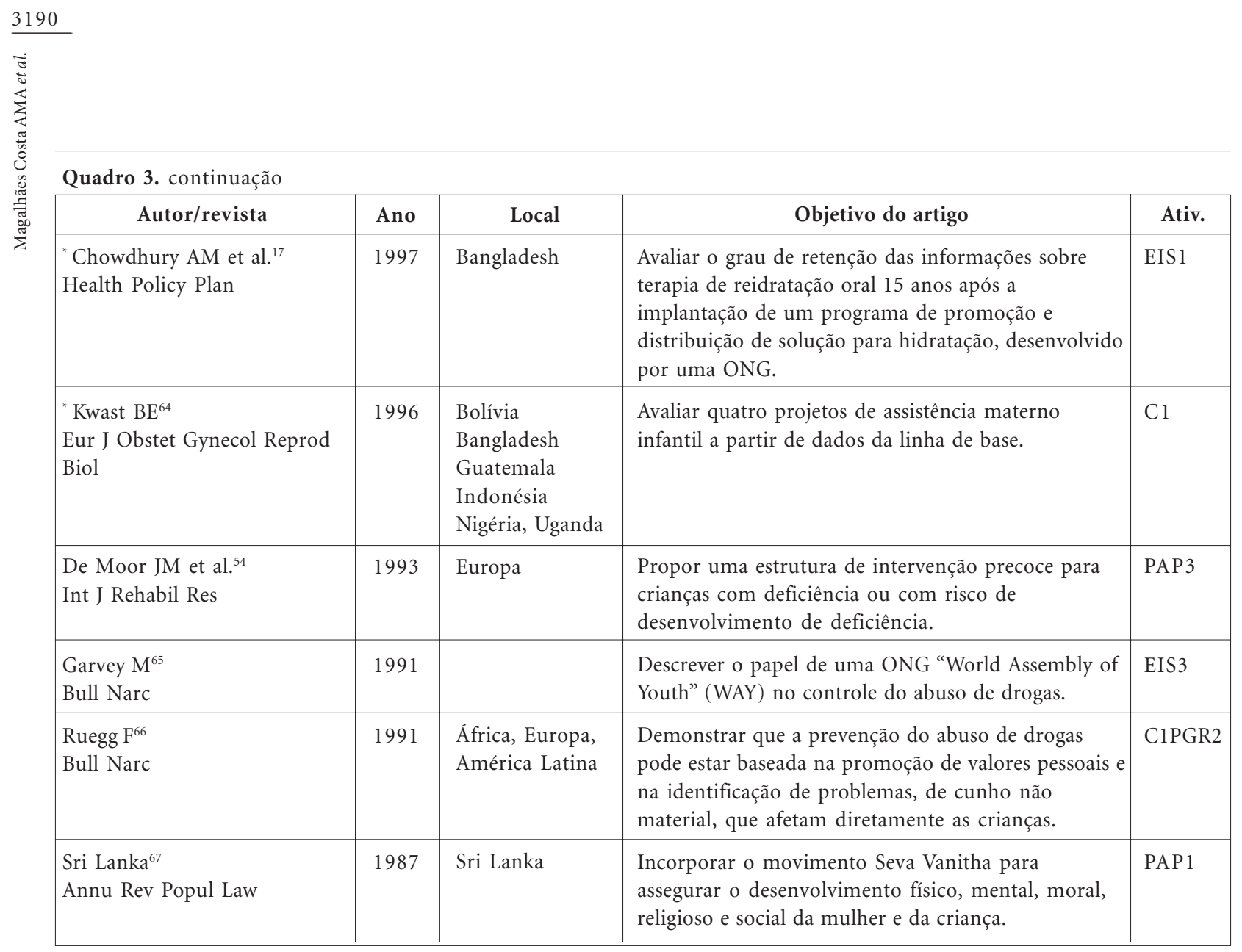

*Artigos que analisam especificamente a atuação de ONG na área da saúde infantil

tre elas, americana, alemã, francesa e inglesa. A ONG que apresentou o maior número de citações foi a ONG brasileira Pastoral da Criança, cujo trabalho foi referido em $3 \operatorname{artigos}^{29,37,40}$. O levantamento do Lilacs contribuiu para esta predominância.

Apenas sete artigos fizeram referência à origem dos recursos utilizados nos projetos. As principais fontes de fomento foram Banco Mundial, Fundo das Nações Unidas para a Criança ${ }^{24}$, United States Agency for International Development (USAID), União Europeia ${ }^{68}$, Child Survival and Health $^{19,20}$, Banco Grameen ${ }^{47}$, Family Health International (FHI) e Official Development Assistance $(\mathrm{ODA})^{51}$, além de consórcios entre estes órgãos e governos locais ${ }^{25}$.

Desse conjunto de artigos, quase dois terços discutiram de modo mais específico aspectos inerentes à atuação da ONG na área da saúde da criança. Em 33\% dos artigos houve considerações sobre a parceria das ONG com instituições de ensino, organismos governamentais, entidades internacionais e serviços de saúde e sua participação em projetos e programas dessas entidades. Em 21\%, havia reflexões associando a atu- ação da ONG aos resultados de intervenções em saúde da criança, atribuindo a estas organizações a efetividade de ações como imunização e introdução da terapia de hidratação oral. Em uma parcela menor de artigos (7\%), a sustentabilidade de projetos e programas assistenciais desenvolvidos por ONG voltados para a saúde da criança foi a preocupação dos autores. Nesses artigos foram feitas proposições de modelos para a viabilização da continuidade dos projetos, apontando, sobretudo, a participação da comunidade beneficiária como fator relevante para a manutenção das práticas incorporadas a partir dos projetos $^{20,29,36,69}$. Os demais artigos (39\%) não faziam considerações específicas sobre a atuação das ONG.

\section{Discussão}

O conjunto de artigos obtidos a partir da consulta com os descritores selecionados aborda o tema das atividades das ONG na área de saúde da criança de forma ampla, provavelmente influenciado pelas definições de ONG adotadas no Medli- 
$\mathrm{ne}^{10}$ e no Lilacs ${ }^{11}$. Para estes bancos de dados, o termo ONG não distingue sociedades, associações ou fundações. Devido a isto, foi possível perceber uma heterogeneidade de perfis entre as organizações apresentadas nas publicações. Por sua vez, ao longo deste trabalho, foram identificadas publicações que abordavam a atuação das ONG na área da saúde da criança e que, no entanto, não foram captadas a partir do levantamento realizado com aqueles descritores. Nesse sentido, tanto um tratamento polissêmico do termo ONG nas bases de dados consultadas, como o fato de que parte dos estudos publicados que investigam a atuação e o papel das ONG na área da saúde infantil não está formalmente indexada com os descritores eleitos, representaram limites à sistematização e análise dessa produção científica sobre esse tema. Além disso, após uma primeira análise dos artigos selecionados, verificou-se que apenas pouco mais da metade deste acervo concentrava-se na análise da atuação ou do papel da ONG na área da saúde infantil. Os demais artigos descreviam práticas voltadas para a saúde infantil, sendo as ONG apenas citadas como um dos atores envolvidos no processo.

Quando se classifica a coletânea científica de acordo com as atividades descritas, chama a atenção a distribuição dos tipos de ações das ONG por estágio de desenvolvimento da região. Ações de assistência à saúde e bem estar, capacitação, educação e informação foram descritas principalmente em países em desenvolvimento, enquanto nos países desenvolvidos as ações se concentraram em atividades relacionadas à participação na agenda programática e política. Devese ter cuidado ao interpretar esse achado. A julgar pela busca realizada nesta pesquisa, ainda são poucos os estudos sobre as ONG que atuam na área da saúde da criança e a maior parte das publicações encontradas referiu-se a países em desenvolvimento, podendo refletir prioridades políticas das instituições de pesquisa e das revistas científicas, sem traduzir a magnitude e a natureza da intervenção das ONG em várias partes do mundo. Por exemplo, Gomes et al. ${ }^{70}$ e Minayo $^{71}$ descreveram a participação de ONG nas discussões de políticas públicas voltadas para a violência na infância e adolescência no Brasil, ressaltando o papel que tiveram na elaboração do Estatuto da Criança e do Adolescente, em 1990, e para o reconhecimento deste grupo social como sujeitos de direito.

Por sua vez, o grande número de ONG envolvidas com atividades assistenciais, educativas e/ou de capacitação em países mais pobres da
África, da Ásia e da América Latina poderia também refletir realidades nacionais onde as instituições públicas ainda têm grande debilidade no que diz respeito à prestação de serviços e os direitos sociais e econômicos ainda não estão garantidos para toda a população. Dirigentes e técnicos de ONG entrevistados por Gomes et al. ${ }^{70}$ referem que assumem tarefas para as quais o Estado tem se mostrado omisso. Em seus estudos, Choy $^{72}$ e Gellert ${ }^{18}$ observaram que as ONG possuem uma capacidade maior do que a do Estado de atingirem áreas carentes e pouco acessíveis. A participação de ONG na distribuição e aplicação de vacinas em regiões longínquas da área rural de Bangladesh contribuiu para uma cobertura maior da população infantil, segundo os estudos de Amin $^{47}$ e Mercer ${ }^{14}$. Estes autores sugeriram que essas ações, de competência do Estado, se mostraram mais eficientes quando desenvolvidas pelas ONG. Da mesma forma, $\mathrm{Khan}^{73} \mathrm{ob}-$ servou que a população alvo de um programa de nutrição em Bangladesh foi mais atingida pela ONG do que pelo órgão governamental, apesar do custo de distribuição do componente nutricional ter sido menor quando realizada pelo governo. Estes achados ratificam a importância do desenvolvimento de mais estudos e uma maior reflexão teórica e política sobre a participação de diferentes atores sociais e institucionais nas ações voltadas para a saúde infantil.

Alguns artigos demonstraram uma preocupação com a sustentabilidade dos projetos implantados. Sarriot ${ }^{19,20}$ se propôs a teorizar essa questão, desenvolvendo um modelo de avaliação de sustentabilidade que preconiza que os projetos e intervenções de ONG devem ter como objetivo a promoção de condições que tornem os indivíduos, a comunidade e as organizações locais aptos a se engajarem em redes de ação e solidariedade em busca da saúde, de modo a diminuir a dependência de recursos incertos (financeiro, humano, técnico e informativo), garantindo a continuidade das ações, mesmo após o encerramento do próprio projeto de intervenção. Uma comunidade bem informada e orientada sobre os procedimentos adotados nos programas é condição importante para a manutenção das ações, uma vez que ao se apropriar do programa, trabalha para sua continuidade ${ }^{20,74}$. As reflexões contidas nesses artigos vão de encontro com aquelas de outros autores que têm estudado associativismo e protagonismo da sociedade civil em outras áreas temáticas ou setoriais. Armani ${ }^{75,76}$ também considera que a sustentabilidade não diz respeito apenas à dimen- 
são financeira de um projeto, mas sim a um conjunto de fatores de desenvolvimento institucional fundamental para o seu sucesso, com destaque para a articulação com os atores locais. Para Cardoso $^{77}$, a sustentabilidade das mudanças introduzidas e fomentadas por projetos sociais depende do desenho de programas que incluam a participação dos beneficiados e promovam o desenvolvimento do capital social que existe em todas as comunidades.

Em alguns dos artigos selecionados, os autores se referem à parceria entre Estado e ONG como uma condição de fortalecimento dos projetos e como fator de facilitação para a sua implantação e manutenção, no entanto, eles não se aprofundam numa análise reflexiva sobre as respectivas responsabilidades e competências ${ }^{29,38}$. A noção de co-responsabilidade tem motivado o estabelecimento de parcerias entre agentes com objetivos comuns apesar das diferenças na lógica de atuação, o que leva a formas de relacionamento que preservam as identidades e as missões institucionais específicas. Nesse sentido, não se pode perder de vista que as diferentes funções e competências dos organismos governamentais e não governamentais têm origem também na natureza distinta dessas instituições. Por exemplo, o controle sobre o conjunto dos recursos socioeconômicos e o poder de decisão sobre sua alocação está fortemente concentrado no Estado - mesmo em seus modelos mais liberais - e, assim, cabe a ele um papel destacado de promoção de justiça social, inclusão e equidade. Entretanto, é cada vez mais reconhecida a necessidade de complementariedade entre as funções e competências governamentais e das organizações da sociedade civil no processo do desenvolvimento social, sem perda da identidade e a missão de cada um dos atores ${ }^{78}$. A colaboração entre ambas esferas promove o protagonismo da sociedade civil em todos os níveis e uma maior transparência para o conjunto das políticas sociais ${ }^{77}$.

A avaliação das ações desenvolvidas pelas ONG e a apresentação pública de seus resultados são fatores importantes para a credibilidade dessas instituições e de seus projetos perante os diversos parceiros e à sociedade civil e, consequentemente, pode contribuir para sua legitimidade e sustentabilidade. $\mathrm{O}$ impacto das ações desenvolvidas por ONG foi objeto de alguns dos artigos revisados. Alguns estudos fizeram avaliação do impacto das intervenções das ONG sobre indicadores de saúde infantil como taxa de mortalidade, taxa de utilização de serviços, co- bertura vacinal, taxa de adesão, nível de incorporação de comportamentos e/ou práticas, e evidenciaram resultados favoráveis. Foram comparados os indicadores de saúde em períodos diferentes, antes e depois da intervenção das $\mathrm{ONG}^{14,16,17,21,64}$ e populações assistidas e não assistidas por essas entidades ${ }^{47}$.

Outro tipo de estudo de impacto encontrado na literatura revisada foi o de custos. $\mathrm{Khan}^{24} \mathrm{com}$ parou os custos da distribuição de vacinas em áreas urbanas de Bangladesh, algumas assistidas por órgãos governamentais e outras por ONG. Os resultados mostraram que a participação da ONG foi mais efetiva tanto na cobertura da população alvo como nos custos dos processos inerentes às ações.

Por ser um tópico importante para a captação de recursos, manutenção de parcerias, viabilidade e sustentabilidade dos projetos, esperavase um maior número de trabalhos que se dedicassem ao impacto das ações. Esta escassez - 12 $\operatorname{artigos}^{14,17,21,24-26,28,35,38,47,52,64}$ - pode ser reflexo das dificuldades em se estabelecer modelos teóricos e metodologias para avaliação das intervenções das ONG na área da saúde da criança. A incorporação de uma prática de avaliação de impacto e custo-efetividade à cultura de gestão das ONG contribuiria para a transparência, o fortalecimento e a confiança dos parceiros e da sociedade.

Gomes et al. ${ }^{70}$ chamam a atenção para o fato de que a multiplicação de ONG não foi acompanhada da adoção de avaliações sistemáticas. Santos e Avritzer ${ }^{79}$ consideram que entre as três instituições que compõem o Estado democrático é o terceiro setor o que menos é submetido a avaliação de desempenho, característica que vulnerabiliza as entidades que o compõem no que diz respeito à sustentabilidade financeira $\mathrm{O}$ funcionamento das ONG depende de fundos públicos e privados voluntários, ou seja não há nenhum tipo de obrigatoriedade legal na continuidade desses financiamentos ${ }^{70}$. Para manter as parcerias, se faz necessária a incorporação de práticas sistemáticas de prestação de contas que devem ser acompanhadas de avaliações metódicas de suas ações e seus impactos. A institucionalização de uma cultura de avaliação das ONG instrumentalizaria as entidades para o monitoramento e gestão dos projetos, contribuiria para demonstrar a relevância de suas ações, ampliar sua legitimidade perante a sociedade e outros atores institucionais e propiciar melhores condições de aprimoramento e de sustentabilidade de seus programas. 
No acervo reunido com os descritores selecionados e os critérios de inclusão e exclusão previamente determinados, observou-se que, na realidade, somente pouco mais da metade dos artigos propunha-se a analisar o papel das ONG na área da saúde infantil, sendo que os demais apenas faziam referências a presença dessas entidades em alguns processos e intervenções. A produção de artigos se deu principalmente nos meios acadêmicos.

Apesar da pequena produção sobre o assunto, os estudos apontaram para a importância da participação de ONG nos programas voltados para a saúde da criança. As ações descritas - orientadas principalmente para a redução da mortalidade infantil e ao acesso à nutrição adequada - vão de encontro a prioridades e diretrizes estabelecidas em consensos internacionais como a Declaração de Alma-Ata ${ }^{80}$, o Encontro Mundial de Cúpula pela Criança ${ }^{81}$ e as Metas do Milênio $^{82}$. As ONG em regiões em desenvolvimento foram as mais estudadas, e as atividades descritas se concentraram na assistência, informação e educação em saúde. O conjunto de artigos pouco esclareceu sobre a participação dessas ONG na elaboração de políticas e na definição de agenda de saúde. Ao contrário, nos estudos realizados em países com níveis mais elevados de desenvolvimento social e econômico sobressai a análise da participação politica das ONG nos processos decisórios de saúde.

Parcerias entre ONG e órgãos governamentais, sustentabilidade das ações e programas implementados, e avaliação de impactos e resultados das intervenções das ONG na área da saú- de infantil sobressaíram como os problemas teóricos e analíticos mais destacados entre aqueles artigos que, em alguma medida, procuraram investigar a atuação e o papel das ONG nessa área. Porém, a maioria dos estudos tratou desses problemas com pouca profundidade. Outras questões teóricas presentes neste campo de estudo tais como o papel dessas organizações como agentes de empoderamento e fortalecimento de cidadania não foram temas muito evidenciados nos artigos estudados.

Esta pesquisa foi um passo para apoiar reflexões sobre avaliação de impacto das ações das ONG na saúde da criança, abrangendo aspectos relacionados aos benefícios diretos sobre a população infantil assim como as repercussões sobre a comunidade que a cerca.

Finalmente, à luz dos resultados deste estudo, sugere-se que a investigação do papel e do impacto das intervenções das ONG na área da saúde da criança é um campo de conhecimento a ser ainda muito explorado. Alguns problemas ou objetos de estudo estão apenas esboçados parcerias, sustentabilidade, avaliação dos programas e prestação de contas de seus resultados - e, um maior conhecimento de outros aspectos já citados referentes à atuação dessas organizações nessa área ainda estão pendentes. Esta pesquisa pode ser considerada como uma contribuição para apoiar esforços e futuras colaborações entre pesquisadores de distintas disciplinas, inserções institucionais e regiões geográficas no sentido de desenvolver marcos teórico-analíticos, metodologias e programas de investigação mais abrangentes sobre os significados e os impactos da atuação da ONG na área da saúde da criança.

\section{Colaboradores}

AMA Magalhães Costa, KS Silva e C Bonan participaram igualmente de todas as etapas de elaboração do artigo. 


\section{Referências}

1. Landim L. "Experiência Militante" História das assim chamadas ONGs. Lusophones 2002; 1:215-239.

2. Ramos S. O papel das ONGs na construção de políticas de saúde: a Aids, a saúde da mulher e a saúde mental. Cien Saude Colet 2004; 9(4):1067-1078.

3. Ckagnazaroff IB, Melo JSC, Reis AM dos. O Papel das ONG's em Processos de Empoderamento de Mulheres na Região Metropolitana de Belo Horizonte. [acessado 2008 mar 4]. Disponível em: http://www. fazendogenero7.ufsc.br/artigos/C/CkagnazaroffMelo-Reis_38_B.pdf

4. Landim L. Para além do mercado e do Estado? Filantropia e cidadania no Brasil. Rio de Janeiro: ISER; 1993. (Série Textos de Pesquisa).

5. Landim L. Associações no Brasil: comentários sobre dados oficiais recentes. Revista Democracia Viva 2005; 28:76-85.

6. Andrade GRB, Vaitsman J. Apoio social e redes: conectando solidariedade e saúde/Social support and network: connecting solidarity and health. Cien Saude Colet 2002; 7(4):925-934.

7. Bonan C. Reflexividade, sexualidade e reprodução. Processos políticos no Brasil e no Chile. [tese]. Rio de Janeiro: Instituto de Filosofia e Ciência Sociais, UFRJ; 2002.

8. Brasil. Ministério da Saúde. AIDS e Sustentabilidade: sobre as ações das organizações da sociedade civil. Brasília-DF. 2001.

9. Brasil. Ministério da Saúde. Sustentabilidade: AIDS e Sociedade Civil em debate. Brasília-DF. 2004.

10. U.S. National Library of Medicine (NLM) PUBMED. [acessado 2008 Apr 16]. Disponível em http:/ /www.ncbi.nlm.nih.gov/PubMed/

11. Literatura latino-americana e do Caribe em Ciências da Saúde - Lilacs. [acessado 2008 abr 16]. Disponível em: http://www.bireme.br

12. Biblioteca Virtual em Saúde. [acessado 2007 abr 16]. Disponível em: http://decs.bvs.br/

13. Stover J, Bollinger L, Walker N, Monasch R. Resource needs to support orphans and vulnerable children in sub-Saharan Africa. Health Policy Plan. 2007; 22(1):21-27.

14. Mercer A, Khan MH, Daulatuzzaman M, Reid J. Effectiveness of an NGO primary health care programme in rural Bangladesh: evidence from the management information system. Health Policy Plan. 2004; 19(4):187-198.

15. Winch PJ, Leban K, Casazza L, Walker L, Pearcy K. An implementation framework for household and community integrated management of childhood illness. Health Policy Plan 2002; 17(4):345-353.

16. Chakraborty S, D'Souza SA, Northrup RS. Improving private practitioner care of sick children: testing new approaches in rural Bihar. Health Policy Plan 2000; 15(4):400-407.

17. Chowdhury AM, Karim F, Sarkar SK, Cash RA, Bhuiya A. The status of ORT (oral rehydration therapy) in Bangladesh: how widely is it used? Health Policy Plan 1997; 12(1):58-66.

18. Gellert GA. Non-governmental organizations in international health: past successes, future challenges. Int J Health Plann Manage 1996; 11(1):19-31.
19. Sarriot EG, Winch PJ, Ryan LJ, Edison J, Bowie J, Swedberg E, Welch R. Qualitative research to make practical sense of sustainability in primary health care projects implemented by non-governmental organizations. Int J Health Plann Manage 2004; 19(1):3-22.

20. Sarriot EG, Winch PJ, Ryan LJ, Bowie J, Kouletio M, Swedberg E, LeBan K, Edison J, Welch R, Pacquel MC. A methodological approach and framework for sustainability assessment in NGO-implemented primary health care programs. Int J Health Plann Manage 2004; 19(1):23-41.

21. Babalola S, Sakolsky N, Vondrasek C, Mounlom D, Brown J, Tchupo JP. The impact of a community mobilization project on health-related knowledge and practices in Cameroon. J Community Health 2001; 26(6):459-477.

22. Cho H, Nadow MZ. Understanding barriers to implementing quality lunch and nutrition education. J Community Health 2004; 29(5):421-435.

23. Baker EJ, Sanei LC, Franklin N. Early Initiation of and exclusive breastfeeding in large-scale community-based programmes in Bolivia and Madagascar. J Health Popul Nutr 2006 24(4):530-539.

24. Khan MM, Khan SH, Walker D, Fox-Rushby J, Cutts F, Akramuzzaman SM. Cost of delivering child immunization services in urban Bangladesh: a study based on facility-level surveys. J Health Popul Nutr 2004; 22(4):404-412.

25. Aikins MK, Fox-Rushby J, D’Alessandro U, Langerock P, Cham K, New L, Bennett S, Greenwood B, Mills A. The Gambian National Impregnated Bednet Programme: costs, consequences and net cost-effectiveness. Soc Sci Med 1998; 46(2):181-191.

26. Valadez JJ, Hage J, Vargas W. Understanding the relationship of maternal health behavior change and intervention strategies in a Nicaraguan NGO network. Soc Sci Med 2005; 61(6):1356-1368.

27. Penny N, Zulianello R, Dreise M, Steenbeek M. Community-based rehabilitation and orthopaedic surgery for children with motor impairment in an African context. Disabil Rehabil 2007; 29(11-12):839843.

28. Senbanjo IO, Adeodu OO, Adejuyigbe EA. Low prevalence of malnutrition in a rural Nigerian community. Trop Doctor 2007; 37(4):214-216.

29. Andrade RD, de Mello DF. Social organizations and governmental institutions: perspectives on partnerships in children's health care through volunteers and the Pastoral da Criança. Rev Esc Enferm USP 2006; 40(1):93-97.

30. Hasanovic M, Sinanovic O, Selimbasic Z, Pajevic I, Avdibegovic E. Psychological disturbances of wartraumatized children from different foster and family settings in Bosnia and Herzegovina. Croat Med J 2006; 47(1):85-94.

31. Andreae MC, Freed GL, Katz SL. Safety concerns regarding combination vaccines. Perspective of select European countries. Hum Vaccin 2005; 1(1):1-5.

32. Gosselin RA, Coppotelli C. A follow-up study of patients with spinal cord injury in Sierra Leone. Int Orthop 2005; 29(5):330-332. 
33. Pinto A, Saeed M, El Sakka H, Rashford A, Colombo A, Valenciano M, Sabatinelli G. Setting up an early warning system for epidemic-prone diseases in Darfur: a participative approach. Disasters 2005; 29(4): 310-322.

34. Stoffel V, Barthelme B, Chague F. Tropical ecopathology: up hill and down dale Buruli ulcer. Sante Publique 2005; 17(2):191-197.

35. Gurgel RQ, da Fonseca JD, Neyra-Castaneda D, Gill GV, Cuevas LE. Capture-recapture to estimate the number of street children in a city in Brazil. Arch Dis Child 2004; 89(3):222-224.

36. de Souza AI, Erdmann AL. The child with a cancer diagnosis: revisiting the way of assistance policies. Rev Gaucha Enferm 2003; 24(1):23-33.

37. Sperandio AMG. Ações estratégicas de promoção da saúde visual: relato de uma experiência. Rev Bras Cresc Desenv Hum 2003; 13(2):59-72.

38. Akerman M. Alunos do $3^{\circ}$ ano CMFMABC. Caracterização da violência doméstica contra crianças na região do $\mathrm{ABCD}$ : em busca de medidas de prevenção. Arq Med ABC 2001; 25(2):44-52.

39. Morikawa M. Upper respiratory infection in acute pediatric care in internal conflict, Kosovo, 1999. J Trop Pediatr 2001; 47(6):379-382.

40. Salvarani MCC, Decesaro MN. A enfermagem inserida no projeto de recuperação do desnutrido. Nursing 2001; 4(38):30-34.

41. Parrilla Rodriguez AM, Gorrin Peralta JJ. Ethical aspects of human lactation and breastfeeding. $P R$ Health Sci J 2000; 19(2):145-151.

42. Ranganathan K, Reddy BV, Kumarasamy N, Solomon S, Viswanathan R, Johnson NW. Oral lesions and conditions associated with human immunodeficiency virus infection in 300 south Indian patients. Oral Dis 2000; 6(3):152-157.

43. Rajeswari R, Balasubramanian R, Muniyandi M, Geetharamani S, Thresa X, Venkatesan P. Socio-economic impact of tuberculosis on patients and family in India. Int J Tuberc Lung Dis 1999; 3(10):869-877.

44. Wegman ME. Foreign aid, international organizations, and the world's children. Pediatrics 1999; 103(3):646-654.

45. Condamine JL, Artigues S, Catherine V, Diagnougou $\mathrm{N}$, Ouoba T. Management of motor disability in rural Sahelian environment. Experience of the center for rehabilitation and devices in Bogande, Burkina Faso. Med Trop (Mars) 1998; 58(4):408-412.

46. Pal DK, Das T, Sengupta S. Comparison of key informant and survey methods for ascertainment of childhood epilepsy in West Bengal, India. Int J Epidemiol 1998; 27(4):672-676.

47. Amin R, Li Y. NGO-promoted women's credit program, immunization coverage, and child mortality in rural Bangladesh. Women Health 1997; 25(1):7187.

48. Leifert H, Murahovsch J. A campanha de acuidade visual. Pediatria Moderna 1997; 33(4):197-204.

49. van der Stuyft P, Delgado E, Sorensen SC. Utilization rates and expenditure for public and private, curative-care services in semi-urban Guatemala. Ann Trop Med Parasitol 1997; 91(2):209-216.
50. Thomas M. The ActionAid disability programmes: experiences in early identification and early intervention. Indian J Pediatr 1992; 59(6):697-700.

51. Crane SF, Carswell JW. A review and assessment of non-governmental organization-based STD/AIDS education and prevention projects for marginalized groups. Health Educ Res 1992; 7(2):175-194.

52. Auer C. Health status of children living in a squatter area of Manila, Philippines, with particular emphasis on intestinal parasitoses. Southeast Asian J Trop Med Public Health 1990; 21(2):289-300.

53. Caraher M, Landon J, Dalmeny K. Television advertising and children: lessons from policy development. Public Health Nutr 2006; 9(5):596-605.

54. De Moor JM, Van Waesberghe BT, Hosman JB, Jaeken D, Miedema S. Early intervention for children with developmental disabilities: manifesto of the Eurlyaid working party. Int J Rehabil Res 1993; 16(1): 23-31.

55. Frenkel LD, Nielsen K. Immunization issues for the $21^{\text {st }}$ century. Ann Allergy Asthma Immunol 2003; 90(Supl. 3):45-52.

56. Potts-Datema W, Smith BJ, Taras H, Lewallen TC, Bogden JF, Murray S. Successful strategies and lessons learned from development of large-scale partnerships of national non-governmental organizations. Promot Educ 2005; 12(3-4):131-137.

57. Forsyth R, Colver A, Alvanides S, Woolley M, Lowe M. Participation of young severely disabled children is influenced by their intrinsic impairments and environment. Dev Med Child Neurol 2007; 49(5):345-349.

58. Goren S. Looking for child psychiatric nursing Vietnam 2005. J Child Adolesc Psychiatr Nurs 2007; 20(3):156-162.

59. Paulson JA, Arneses SJ. The use of the internet for children's health and the environment. Pediatr Clin N Am 2007; 54(1):135-153.

60. Lechting A, Gross R, Vivanco OA, Gross U, Romaña DL de. Lessons learned from the scaling-up of weekly multimicronutrient supplementation program in the Integrated Food Security Program (PISA). Food Nutr Bull 2006; 27(Supl. 4):160-165.

61. Brusamolino E, Maffi G. Health cooperation in an hospital of a rural area of Ivory Coast: analysis of the priorities and of the different levels at which cooperation can take place. Crit Rev Oncol Hematol 2004; 49(1):43-51.

62. Balinska MA...Vaccination in tomorrow's society. Lancet Infect Dis 2003; 3(7):443-447.

63. Pick S, Givaudan M, Brown J. Quietly working for school-based sexuality education in Mexico: strategies for advocacy. Reprod Health Matters 2000; 8(16):92-102.

64. Kwast BE. Reduction of maternal and perinatal mortality in rural and peri-urban settings: what works? Eur J Obstet Gynecol Reprod Biol 1996; 69(1): 47-53.

65. Garvey M. Information and alternatives: the role of a youth non-governmental organization in drug abuse control. Bull Narc 1991; 43(1):29-33. 
66. Ruegg F. The International Catholic Child Bureau and drug abuse: contributions to drug abuse prevention by a non-governmental organization concerned with children. Bull Narc 1991; 43(1):9-15.

67. Lanka Sri. Seva Vanitha Movement of Sri Lanka (Incorporation). (Act No 10, 1987), 10 Mar 1987. Annu Rev Popul Law 1987; 14:131.

68. Ahmed A, Edward A, Burnham G. Health indicators for mothers and children in rural Herat Province, Afghanistan. Prehospital Disaster Med 2004; 19(3):221-225.

69. Rocha PR. Estação cidadania - uma experiência no atendimento de crianças e adolescentes em situação de risco. Rev Ter Ocup Univ São Paulo 2006; 17 (1):37-41.

70. Gomes R, Minayo MCS, Assis SG de, Njaine K, Schenker M. Êxitos e limites na prevenção da violência: estudo de caso de nove experiências brasileiras. Cien Saude Colet 2007; 11(Supl.):1291-1302.

71. Minayo, MCS. A inclusão da violência na agenda da saúde: trajetória histórica. Cien Saude Colet 2007; 11(Supl.):1259-1267

72. Choy R, Trevor D. The role of non-governmental organizations in supporting and integrating interventions to improve child health. PNG Med J 2000; 43(1-2):76-81.

73. Khan MM, Ahmed S. Relative efficiency of government and non-government organizations in implementing a nutrition intervention programme - a case study from Bangladesh. Public Health Nutrition 2003; 6(1):19-24.

74. Akukwe C, The Growing Influence of Non Governmental Organizations (NGOs) in International Health: Challenges and Opportunities. J Roy Soc Health 1998; 118(2):107-115.

75. Armani, D. O desenvolvimento institucional como condição de sustentabilidade das ONG no Brasil. Ministéro da Saúde 2001; 17-33 [acessado 2008 maio 14]. Disponível em: http://bvsms.saude.gov.br

76. Armani D. Sustentabilidade: desafio democrático. Secretaria de Vigilância em Saúde, Programa Nacional de DST e Aids. - Brasília: Ministério da Saúde 2004. [acessado 2008 mar 3] 9-14. Disponível em: http:// bvsms.saude.gov.br/bvs/publicacoes/sustentabilidade. pdf\#page $=76$
77. Cardoso, R. Sustentabilidade, o desafio das políticas sociais no século. São Paulo em Perspectiva 2004; 18(2):42-48.

78. Mattos SMSN, Drummond JA. O terceiro setor como executor de políticas públicas: ONG's ambientalistas na baía de Guanabara (1990-2001) Rev Sociol Polit 2005; 24:177-192.

79. Santos BS, Avritzer L. Para ampliar o cânone democrático. In: Santos BS, organizador. Democratizar a democracia: os caminhos da democracia participativa. Rio de Janeiro: Editora Civilização Brasileira; 2002. p. 39-82.

80. World Health Organization (WHO). UNICEF. Primary Health Care. In: International Conference on Primary Care Alma-Ata; 1978; USSR.

81. Encontro Mundial de Cúpula pela Criança 1990. Declaração Mundial sobre a sobrevivência, a proteção e o desenvolvimento da criança e Plano de Ação para a implementação da Declaração Mundial sobre a sobrevivência, a proteção e o desenvolvimento da criança nos anos 90. Brasília: UNICEF; [1990].

82. World Health Organization (WHO). Objetivos de desarrollo del milenio [acessado 2008 jan 25]. Disponível em: http://www.who.int/mdg/goals/goal4/ context/en/index.html

Artigo apresentado em 13/08/2008

Aprovado em 04/11/2008

Versão final apresentada em 28/11/2008 